\title{
Bioinformatic Analysis for Potential Biomarkers and Therapeutic Targets of T2DM-related MI
}

\author{
Chan $\mathrm{Li}^{1}$ \\ Zhaoya Liu $^{2}$ \\ 'Department of Cardiovascular Medicine, \\ Xiangya Hospital, Central South \\ University, Changsha, Hunan, People's \\ Republic of China; ${ }^{2}$ Department of \\ Geriatrics, Third Xiangya Hospital, \\ Central South University, Changsha, \\ Hunan, People's Republic of China
}

Background: Type 2 diabetes mellitus (T2DM), a major risk factor of coronary heart disease, is associated with an approximately twofold increase in the risk of myocardial infarction (MI). We studied co-expressed genes to demonstrate relationships between DM and MI and revealed the potential biomarkers and therapeutic targets of T2DM-related MI. Methods: DM and MI-related differentially expressed genes (DEGs) were identified by bioinformatic analysis, Gene Expression Omnibus (GEO) datasets GSE42148 and GSE61144 of MI patients, and the normal control and GSE26168 and GSE15932 of DM patients and normal controls, respectively. Further target prediction and network analysis method were used to detect protein-protein interaction (PPI) networks, gene ontology (GO) terms, and pathway enrichment of DEGs. Co-expressed DEGs of T2DM-related MI were analyzed as well.

Results: We identified 210 upregulated and 127 downregulated DEGs in T2DM, as well as 264 upregulated and 242 downregulated DEGs in MI. Eighteen upregulated and four downregulated DEGs were identified as co-DEGs of T2DM and MI. Functional analysis revealed that T2DM-related DEGs were mostly enriched in the viral process and ubiquitin-mediated proteolysis, while MI-related DEGs were mostly enriched in protein phosphorylation and TNF signaling pathway. MPO, MMP9, CAMP, LTF, AZU1, DEFA4, STAT3, and PECAM1 were recognized as the hub genes of the co-DEGs with acceptable diagnostic values in T2DM and MI datasets. Adenosine receptor agonist IB-MECA was predicted to be a potential drug for T2DM-related MI with the highest CMap connectivity score.

Conclusion: Our study identified that the co-DEGs of MPO, MMP9, CAMP, LTF, AZU1, DEFA4, STAT3, and PECAM1 are significantly associated with novel biomarkers involved in T2DM-related MI. However, more experimental research and clinical trials are demanded to verify our results.

Keywords: myocardial infarction, diabetes mellitus, Gene Expression Omnibus, differentially expressed genes, protein-protein interaction

\section{Introduction}

Diabetes mellitus (DM), an increasingly common metabolic disorder, is a leading cause of morbidity and mortality and creates a significant public health burden. ${ }^{1}$ The latest number of diabetes patients is expected to increase to 642 million by 2040 , which will further increase the economic burden on society. ${ }^{2}$ The economic burden associated with DM is primarily attributed to both macrovascular and microvascular dysfunction and complications. ${ }^{3}$ Abundant studies indicated that T2DM is associated with a substantially increased risk of all-cause death, especially myocardial infarction, peripheral artery diseases, retinopathy, end-stage renal disease, and neuropathy. ${ }^{4-6}$
Correspondence: Zhaoya Liu

Third Xiangya Hospital, Central South

University, No. 138 Tongzipuo Road,

Changsha, Hunan, 4I0013, People's

Republic of China

Email liuzhaoya@csu.edu.cn 
It is well-known that myocardial infarction (MI) is another common problem resulting in cardiovascular events, including cardiac death and heart failure. ${ }^{7}$ Diabetes is an independent risk factor of MI. In fact, MI is the leading cause of death among patients with DM, and individuals with DM are at increased risk of recurrent cardiovascular events and mortality after MI. ${ }^{8}$ Clinical studies indicated that mortality rates post-MI are twofold higher in DM than non-DM patients, and the rate of cardiovascular death is increased by 4.4-fold in DM alone without other traditional cardiovascular factors compared with non-DM in the same age groups. ${ }^{9}$ The FAST-MI study suggested that patients with DM had a $70 \%$ increased risk of being hospitalized for nonfatal heart failure in the year following MI (adjusted hazard ratio 1.70), which shed much-needed light on the impact of DM on heart failure after acute MI. ${ }^{10}$ Partial studies revealed some molecular pathways including decreased mitophagy and metabolic dysfunction that contributes to the death from MI related to DM. ${ }^{11,12}$ However, the related genetics research for finding prognostic markers is still limited and demands further exploration.

In the present study, two gene expression profiles for MI and T2DM were downloaded and analyzed. Differentially expressed genes (DEGs) between normal controls and MI or T2DM were found, and the co-expressed DEGs of the two diseases were obtained as well. Gene ontology (GO), Kyoto Encyclopedia of Genes and Genomes (KEGG) and proteinprotein interaction (PPI) analysis were conducted to detect the hub genes. Consequently, hub DEGs and the related molecular pathways may be better to understand the relationship between T2DM and MI.

\section{Methods}

\section{Data Resources}

Gene expression profile data of peripheral blood samples from T2DM and MI patients were searched in the Gene Expression Omnibus (GEO) database (https://www.ncbi. nlm.nih.gov/geoprofiles/). GSE26168 with nine T2DM patients and eight controls, GSE15932 with eight T2DM patients and eight controls, GSE42148 with blood samples of $13 \mathrm{MI}$ patients and 11 controls, and GSE61144 with 14 MI patients and 10 controls were downloaded for analyses in this study. The detailed information of these datasets was shown in Table 1. Since these datasets were obtained from public databases, ethical approval was not necessary.

\section{Identification of DEGs}

To preprocess the data and screen DEGs between patients and controls, limma package in R-platform (R-project.org) was used and statistical difference was defined as $p$-value $<0.05$. Fold change (FC) was identified by calculating the ratio of gene expression level in patients and controls. Logarithmic operation with 2 as a base number was adopted to help with the comparison. Those with $\log _{2} \mathrm{FC}<0$ were considered as downregulated genes, and $\log _{2} \mathrm{FC}>0$ were considered as upregulated genes. In addition, heat maps and Venn diagrams for DEGs were created via R-platform. The relative codes were provided as Appendix 1.

\section{Functional and Pathway Enrichment Analyses}

To better understanding the function of DEGs, DAVID software (https://david.ncifcrf.gov/) was used to conduct gene ontology (GO), including biological process (BP), cellular component (CC), and molecular function (MF), and KEGG pathway enrichment analysis of T2DM- and MI-DEGs. A $p$-value of $<0.05$ was recognized as significantly enriched.

\section{Protein-protein Interaction (PPI) Analysis} PPI networks of T2DM-, MI- and co-DEGs were established via the online tool STRING (http://string-db.org/) and analytic results were downloaded with a confidence score $>0.40$. Hub genes and core modules, which included a cluster of proteins with the highest degree of interaction in PPI network, were selected and visualized by Cytoscape (http://cytoscape.org/) subsequently.

Table I Details of Datasets

\begin{tabular}{|c|c|c|c|c|c|c|c|}
\hline Disease & GEO ID & Platform & Organism & Experiment Type & Samples (Case vs Control) & Country & Year \\
\hline \multirow[t]{2}{*}{ T2DM } & GSE26168 & GPL6883 & Homo sapiens & Expression profiling by array & 9 vs 8 & Singapore & 2010 \\
\hline & GSEI5932 & GPL570 & Homo sapiens & Expression profiling by array & 8 vs 8 & China & 2012 \\
\hline \multirow[t]{2}{*}{ MI } & GSE42 I 48 & GPLI 3607 & Homo sapiens & Expression profiling by array & 13 vs II & India & 2012 \\
\hline & GSE6I I 44 & GPL6106 & Homo sapiens & Expression profiling by array & 14 vs 10 & South Korea & 2015 \\
\hline
\end{tabular}

Abbreviations: T2DM, type 2 diabetes mellitus; MI, myocardial infarction. 


\section{Conduction of Receiver Operating Characteristic (ROC) Curves}

Graph Prism 6 was applied to conduct ROC curves and calculate the area under the curve (AUC) of the hub genes of co-DEGs, which represented the diagnostic efficiency of genes. A $p$-value of $<0.05$ was regarded as statistically significant.

\section{Prediction of Potential Drugs for T2DM-related MI}

Upregulated co-DEGs were submitted to L1000 platform (https://clue.io/), and potential drugs for T2DM-related MI were predicted. CMap connectivity score $>90$ or CMap connectivity score <-90 was considered to be the standard for recognizing potentially effective drugs.

\section{Results}

\section{Identification of DEGs}

When comparing the blood samples of T2DM patients and normal controls, 1870 upregulated and 2156 downregulated genes were identified in GSE26168, while 3188 upregulated and 1400 downregulated genes were identified in GSE15932. As shown in Figure 1, 210 common upregulated and 127 common downregulated genes of GSE26168 and GSE15932 were recognized as T2DM-DEGs. As for MI patients compared with controls, 1910 upregulated and 1847 downregulated genes were identified in GSE42148, while 2046 upregulated and 3170 downregulated genes were identified in GSE61144. In addition, 264 common upregulated and 242

A

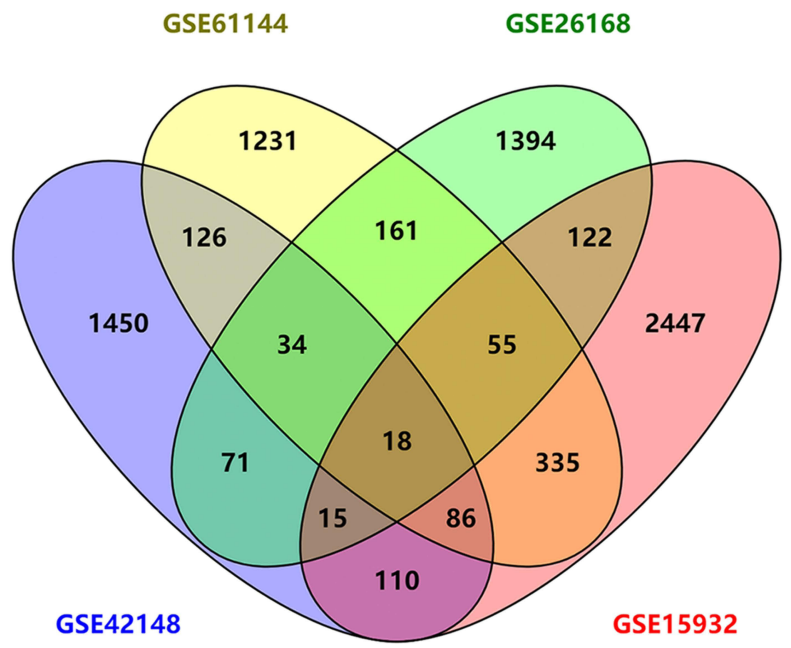

common downregulated genes were recognized as MIDEGs. Subsequently, co-DEGs were analyzed by comparing T2DM-DEGs with MI-DEGs, where 18 upregulated DEGs and four downregulated DEGs were identified (Figure 1). T2DM, MI- and co-DEGs were listed in Supplement Table 1, while heatmaps of the DEGs with largest fold change were presented in Supplement Figures 1 and 2.

\section{Functional Enrichment Analysis}

GO analysis revealed that T2DM DEG-related biological processes (BP) mainly enriched in viral process ( $p$-value $=0.0001)$, proteasome-mediated ubiquitin-dependent protein catabolic process ( $p$-value $=0.0004)$, and positive regulation of peptidyl-serine phosphorylation ( $p$-value $=0.0091$ ). Changes in GO cellular components (CC) mainly included extracellular exosome $(p$-value $=0.0008), \quad$ nucleoplasm ( $p$-value $=0.0025)$, and cytosol ( $p$-value $=0.0047)$. There was significant correlation in GABA receptor binding ( $p$-value $=0.0019)$, protein binding $(p$-value $=0.0043)$, and ubiquitin conjugating enzyme activity ( $p$-value $=0.0153$ ) in relation to molecular function (MF) (Figure 2A).

With respect to MI-DEGs, the BP terms of protein phosphorylation ( $p$-value $<0.0001)$, inflammatory response ( $p$-value $=0.0001)$ and mitochondrial translational elongation ( $p$-value $=0.0003$ ) were significantly enriched. In the CC category, enriched GO terms were associated with mitochondrial large ribosomal subunit ( $p$-value $=0.0001)$, cytosol $(p$-value $=0.0005)$, and mitochondrial inner membrane ( $p$-value $=0.0007)$. In addition, the terms of protein binding ( $p$-value $<0.0001$ ),

B

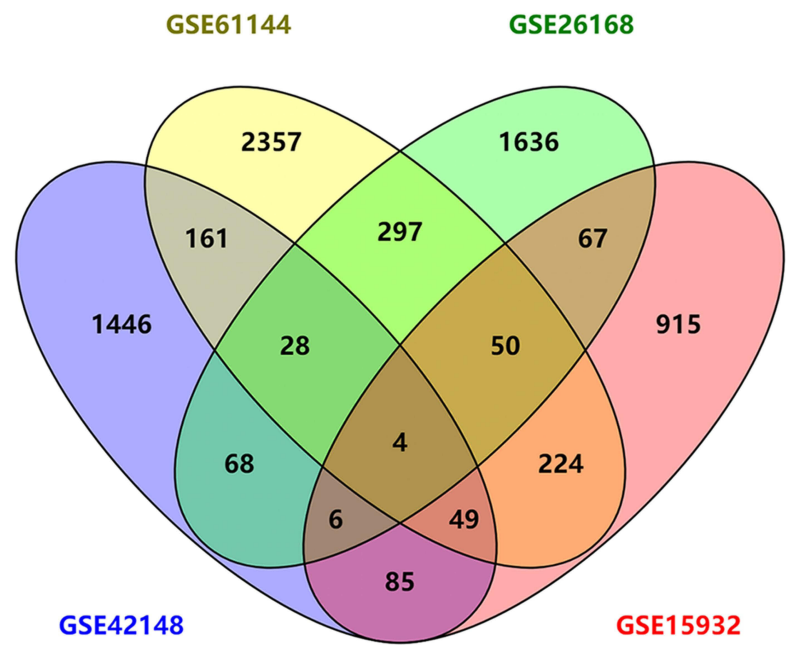

Figure I Venn diagrams for (A) upregulated differentially expressed genes (DEGs); (B) downregulated DEGs. 
A

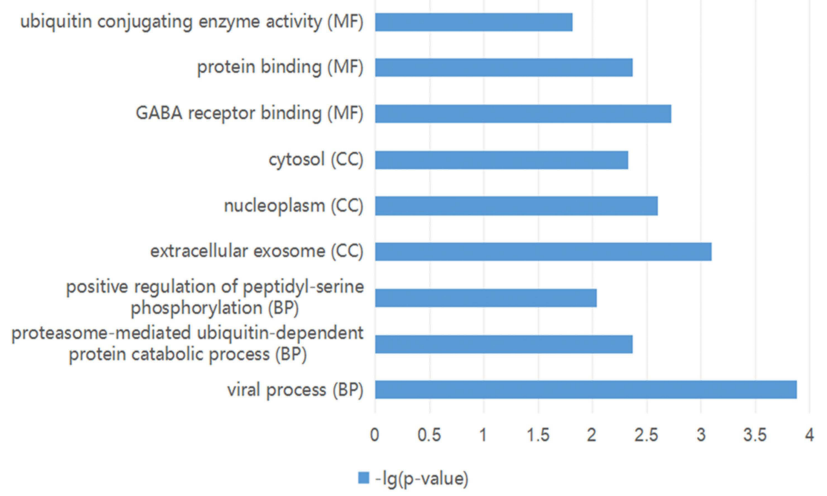

B

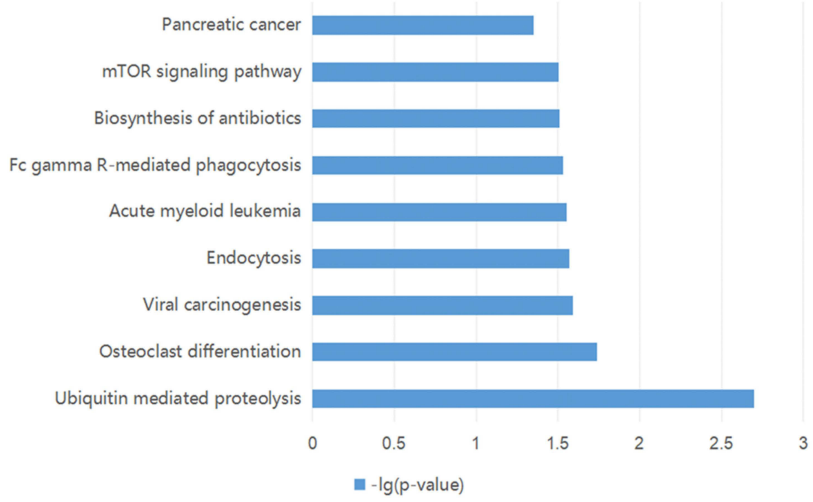

Figure 2 Functional analyses of differentially expressed genes (DEGs) in type 2 diabetes mellitus (T2DM). (A) The top three enriched gene ontology (GO) terms in biological process (BP), cellular component (CC) and molecular function (MF); (B) the top nine enriched Kyoto Encyclopedia of Genes and Genomes (KEGG) pathways.

protein serine/threonine kinase activity ( $p$-value $<0.0001)$, and protein kinase activity ( $p$-value $=0.00026)$ related MF were enriched (Figure 3A).

KEGG pathway analysis was also performed and T2DMDEGs were majorly enriched in the pathway of ubiquitinmediated proteolysis $(p$-value $=0.0020)$ osteoclast differentiation $(p$-value $=0.0184)$, and viral carcinogenesis ( $p$-value $=0.0256)$, while MI-DEGs had a strong correlation with TNF signaling pathway ( $p$-value $=0.0051$ ), tuberculosis ( $p$-value $=0.0074)$ and VEGF signaling pathway ( $p$-value $=0.0101)$, which were illustrated in Figures $2 \mathrm{~B}$ and $3 \mathrm{~B}$.

\section{PPI Network Analysis}

We identified 253, 421, and 12 nodes, and 572, 1377, and 20 edges from PPI network of T2DM-, MI- and coDEGs, respectively. UBE3A, CDC23, $W W P 1, S M U R F 2$, HACE1, CUL5, SIAH2, KBTBD8, CDC34, and UBE2H

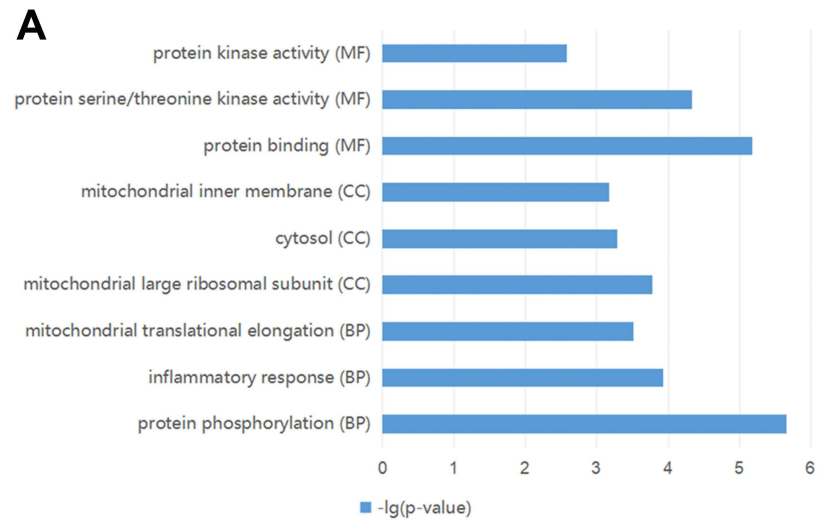

were recognized as hub genes in T2DM-DEGs (Figure 4), while AKT1, STAT3, TLR8, TLR2, MMP9, PTGS2, CXCL1, CCL5, MPO, and CD4OLG were the top 10 hub genes of MI-DEGs (Figure 5). With respect to co-DEGs, $M P O, M M P 9, C A M P, L T F, A Z U 1, D E F A 4$, STAT3, and PECAM1 had much abundant connectivity (Figure 6). Details of the hub genes were presented in Table 2.

\section{Diagnostic Value of Hub Genes of Co-DEGs}

ROC curves were constructed to validate the diagnostic value of the hub genes of the co-DEGs in T2DM and MI datasets, respectively. As shown in Figure 7, The AUC for AZU1, CAMP, DEFA4, LTF, MMP9, MPO, PECAM1, and STAT3 in T2DM patients were 0.986 (0.943-1.030), 0.861 (0.677-1.045), 0.931 (0.807-1.054), 0.903 (0.755-1.051), 0.986 (0.943-1.030), 0.958 (0.866-1.051), 0.833 (0.637-

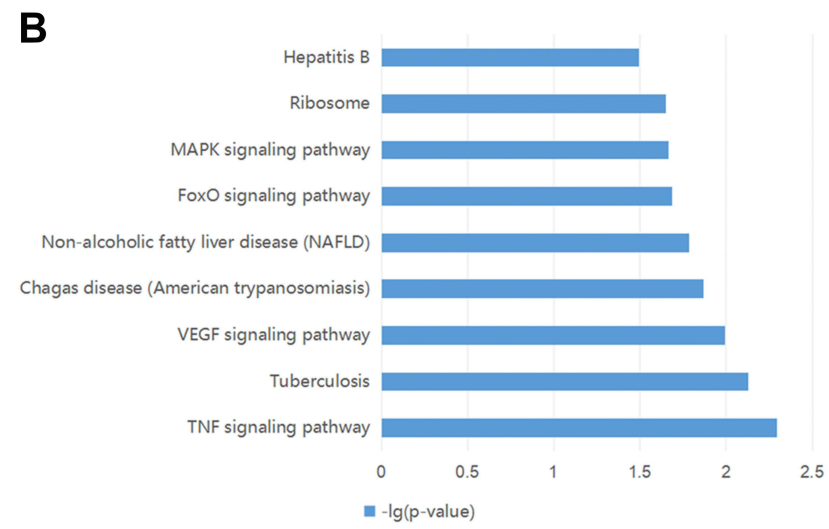

Figure 3 Functional analyses of differentially expressed genes (DEGs) in myocardial infarction (MI). (A) The top three enriched gene ontology (GO) terms in biological process (BP), cellular component (CC) and molecular function (MF); (B) the top nine enriched Kyoto Encyclopedia of Genes and Genomes (KEGG) pathways. 


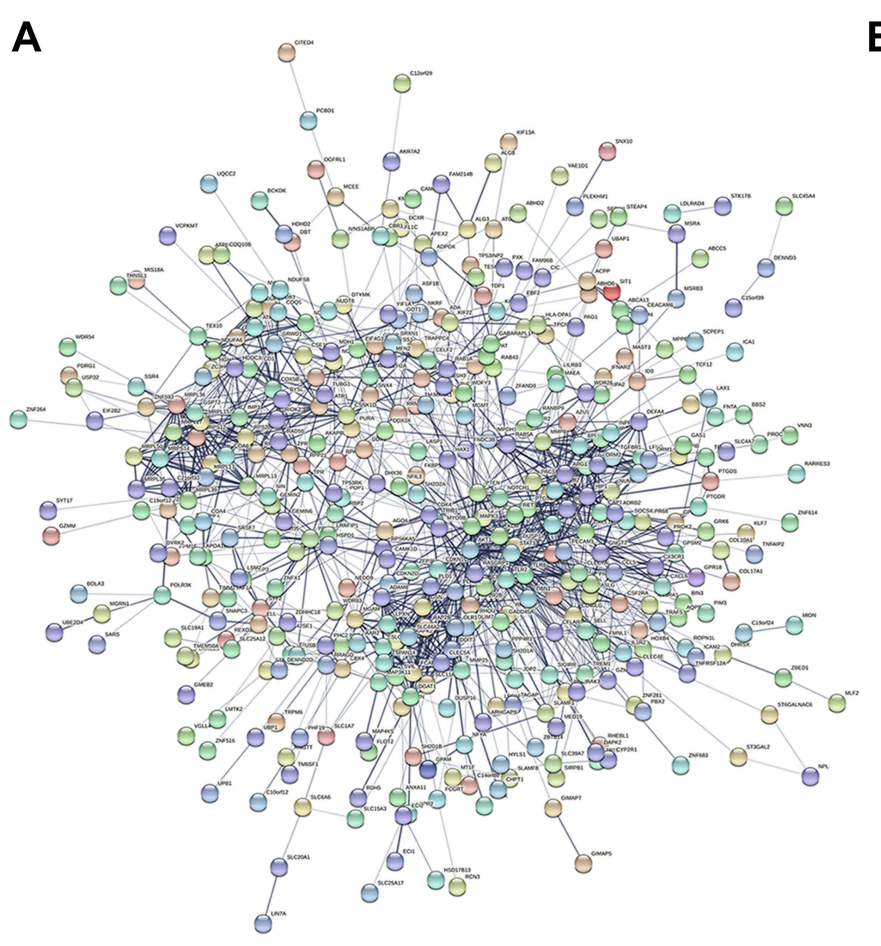

\section{B}

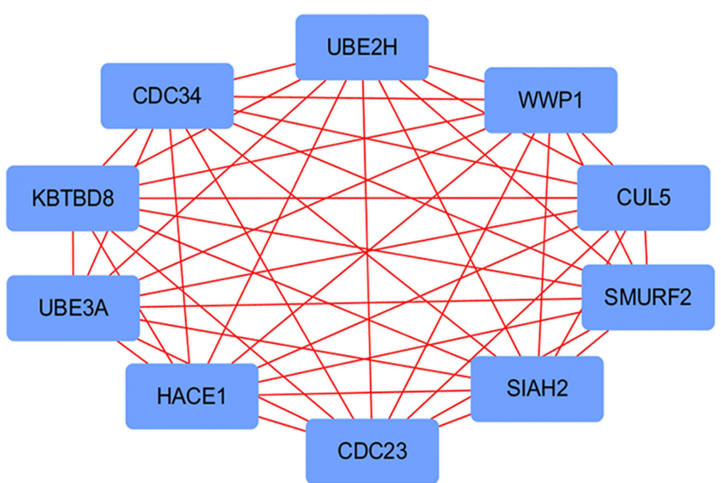

Figure 4 Protein-protein interaction (PPI) analysis (A) and hub genes; (B) of type 2 diabetes mellitus (T2DM) related differentially expressed genes (DEGs).

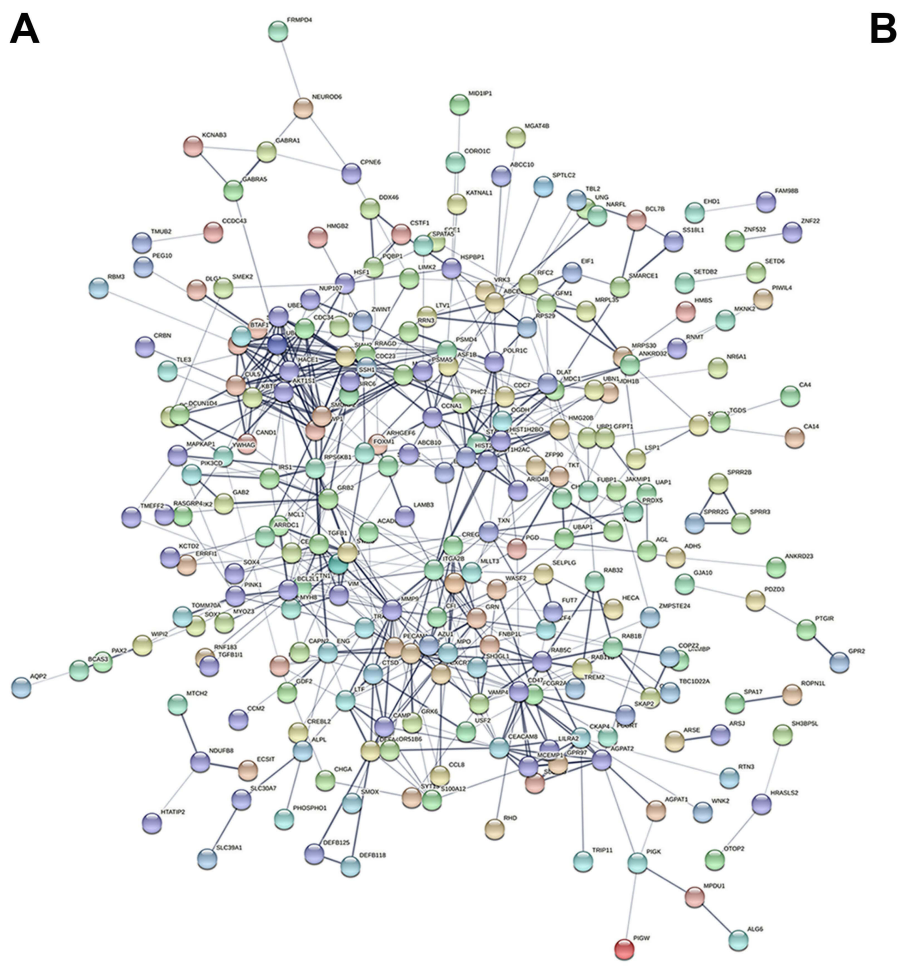

B

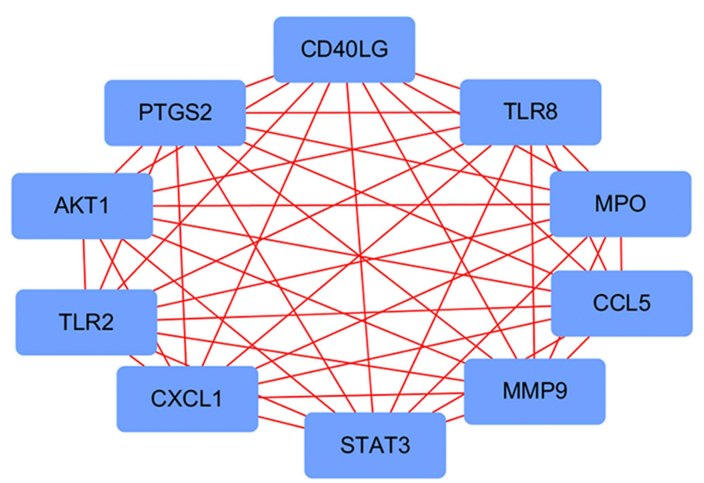

Figure 5 Protein-protein interaction (PPI) analysis (A) and hub genes; (B) of myocardial infarction (MI) related differentially expressed genes (DEGs).

$1.030)$, and 0.819 (0.596-1.043), respectively. As for MI patients, the AUC for these co-DEGs were 0.757 ( 0.558 0.957), 0.932 (0.827-1.038), 0.829 (0.666-0.991), 0.800
(0.621-0.979), 0.929 (0.829-1.028), 0.814 (0.641-0.988), 0.821 (0.655-0.988), and $0.836(0.674-0.998)$, respectively (Figure 8). 


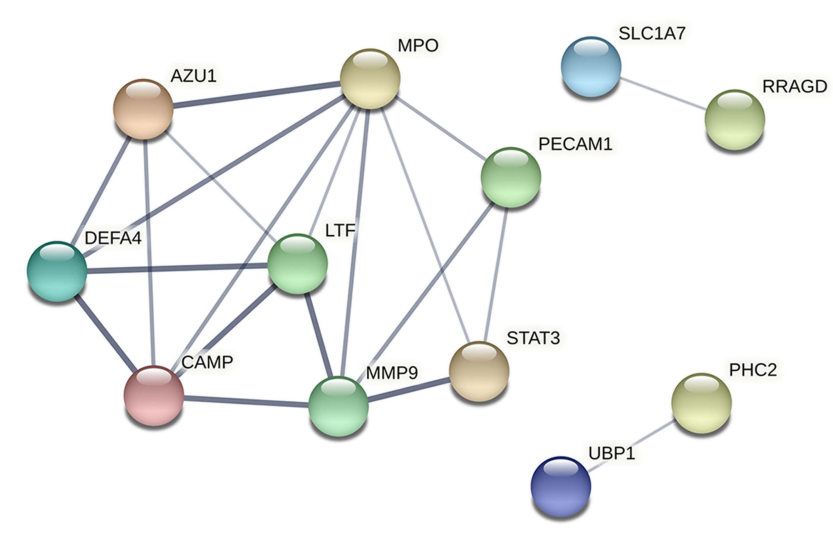

Figure 6 Protein-protein interaction (PPI) analysis of co-expressed differentially expressed genes (co-DEGs).

\section{Prediction of Potential Drugs for T2DM-related MI}

Potential drugs for T2DM-related MI were analyzed by uploading the 18 upregulated co-DEGs to L1000 platform, while the top 10 and bottom 10 chemical compounds ranked by CMap connectivity score were listed in Table 3 .

\section{Discussion}

Type 2 diabetes mellitus (T2DM) and myocardial infarction (MI) represent two major health burdens with steadily increasing prevalence and mortality. ${ }^{13}$ T2DM is a chronic disorder that is characterized by hyperglycemia and insulin resistance. It has been recognized as an independent risk factor for cardiovascular disease including atherosclerosis, myocardial infarction, nephropathy. ${ }^{14}$ Much evidence has demonstrated that the pathogenesis of diabetes mellitus and atherosclerosis have a strong correlation, while the mechanisms of the connection between diabetes and atherosclerosis are not fully elucidated. The production of advanced glycation end-products (AGEs), increased oxidative stress, endothelium injury, inflammation, dyslipidemia were investigated to contribute to diabetes mellitus to

Table 2 Hub Genes Identified in PPI Network

\begin{tabular}{|c|c|c|c|}
\hline Groups & Gene & Description & Degree of Connectivity \\
\hline T2DM DEGs & $\begin{array}{l}\text { UBE3A } \\
\text { CDC23 } \\
\text { WWPI } \\
\text { SMURF2 } \\
\text { HACEI } \\
\text { CUL5 } \\
\text { SIAH2 } \\
\text { KBTBD8 } \\
\text { CDC34 } \\
\text { UBE2H }\end{array}$ & $\begin{array}{l}\text { Ubiquitin protein ligase E3A } \\
\text { Cell Division cycle } 23 \\
\text { WW domain containing E3 ubiquitin protein ligase I } \\
\text { SMAD specific E3 ubiquitin protein ligase } 2 \\
\text { HECT domain and ankyrin repeat containing E3 ubiquitin protein ligase I } \\
\text { Cullin } 5 \\
\text { Siah E3 ubiquitin protein ligase } 2 \\
\text { Kelch repeat and BTB domain containing } 8 \\
\text { Cell division cycle } 34 \text {, ubiqiutin conjugating enzyme } \\
\text { Ubiquitin conjugating enzyme E2 H }\end{array}$ & $\begin{array}{l}19 \\
15 \\
14 \\
14 \\
13 \\
13 \\
12 \\
12 \\
12 \\
11\end{array}$ \\
\hline MI DEGs & $\begin{array}{l}\text { AKTI } \\
\text { STAT3 } \\
\text { TLR8 } \\
\text { TLR2 } \\
\text { MMP9 } \\
\text { PTGS2 } \\
\text { CXCLI } \\
\text { CCL5 } \\
\text { MPO } \\
\text { CD4OLG }\end{array}$ & $\begin{array}{l}\text { AKT serine/threonine kinase I } \\
\text { Signal transducer and activator of transcription } 3 \\
\text { Toll like receptor } 8 \\
\text { Toll like receptor } 2 \\
\text { Matrix metallopeptidase } 9 \\
\text { Prostaglandin-endoperoxide synthase } 2 \\
\text { C-X-C motif chemokine ligand I } \\
\text { C-C motif chemokine ligand } 5 \\
\text { Myeloperoxidase } \\
\text { CD40 ligand }\end{array}$ & $\begin{array}{l}64 \\
48 \\
36 \\
36 \\
35 \\
34 \\
32 \\
27 \\
26 \\
21\end{array}$ \\
\hline Co-DEGs & $\begin{array}{l}\text { MPO } \\
\text { MMP9 } \\
\text { CAMP } \\
\text { LTF } \\
\text { AZUI } \\
\text { DEFA4 } \\
\text { STAT3 } \\
\text { PECAMI }\end{array}$ & $\begin{array}{l}\text { Myeloperoxidase } \\
\text { Matrix metallopeptidase } 9 \\
\text { Cathelicidin antimicrobial peptide } \\
\text { Lactotransferrin } \\
\text { Azurocidin I } \\
\text { Defensin alpha } 4 \\
\text { Signal transducer and activator of transcription } 3 \\
\text { Platelet and endothelial cell adhesion molecule I }\end{array}$ & $\begin{array}{l}7 \\
5 \\
5 \\
5 \\
4 \\
4 \\
3 \\
3\end{array}$ \\
\hline
\end{tabular}

Abbreviations: PPI, protein-protein interaction; DEGs, differentially expressed genes; T2DM, type 2 diabetes mellitus; MI, myocardial infarction. 
A

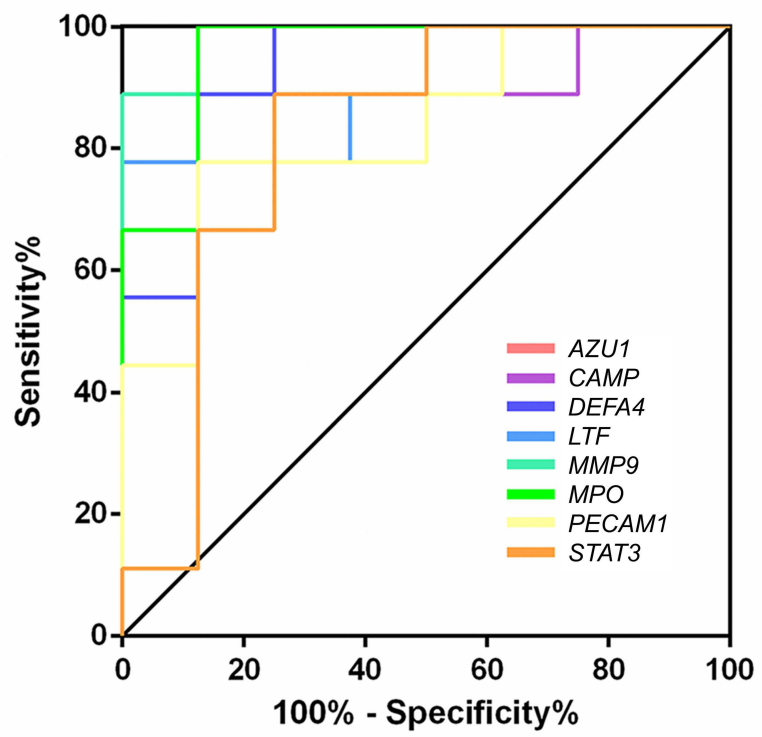

B

\begin{tabular}{ccc}
\hline Gene & $\boldsymbol{p}$-value & AUC $(95 \% \mathrm{Cl})$ \\
\hline AZU1 & 0.001 & $0.986(0.943-1.030)$ \\
CAMP & 0.012 & $0.861(0.677-1.045)$ \\
DEFA4 & 0.003 & $0.931(0.807-1.054)$ \\
LTF & 0.005 & $0.903(0.755-1.051)$ \\
MMP9 & 0.001 & $0.986(0.943-1.030)$ \\
MPO & 0.002 & $0.958(0.866-1.051)$ \\
PECAM1 & 0.021 & $0.833(0.637-1.030)$ \\
STAT3 & 0.027 & $0.819(0.596-1.043)$ \\
\hline
\end{tabular}

Figure 7 Diagnostic value of hub genes of the co-expressed differentially expressed genes (co-DEGs) in type 2 diabetes mellitus (T2DM). (A) ROC curves; (B) specific value of diagnosis efficiency.

A

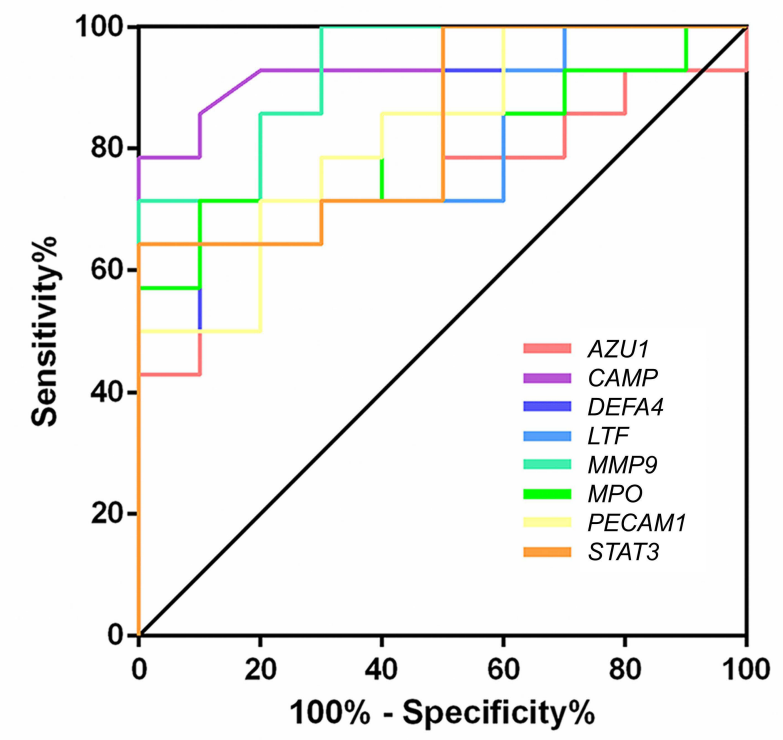

B

\begin{tabular}{ccc}
\hline Gene & $\boldsymbol{p}$-value & AUC $(95 \% \mathrm{Cl})$ \\
\hline AZU1 & 0.035 & $0.757(0.558-0.957)$ \\
CAMP & 0.000 & $0.932(0.827-1.038)$ \\
DEFA4 & 0.007 & $0.829(0.666-0.991)$ \\
LTF & 0.014 & $0.800(0.621-0.979)$ \\
MMP9 & 0.000 & $0.929(0.829-1.028)$ \\
MPO & 0.010 & $0.814(0.641-0.988)$ \\
PECAM1 & 0.008 & $0.821(0.655-0.988)$ \\
STAT3 & 0.006 & $0.836(0.674-0.998)$ \\
\hline
\end{tabular}

Figure 8 Diagnostic value of hub genes of the co-expressed differentially expressed genes (co-DEGs) in myocardial infarction (MI). (A) ROC curves; (B) specific value of diagnosis efficiency.

atherosclerosis. ${ }^{15}$ Although various drugs, adequate glycemic control, and reduction of risk factors had been applied, the complications including atherosclerosis are still inevitable in patients with diabetes mellitus. ${ }^{16}$ In addition, previous studies also showed that mortality rates postMI are much higher in DM patients than the non-DM groups. ${ }^{17,18}$
Therefore, it is necessary to explore the mechanism and discover the novel target to prevent T2DM-related MI.

In this study, we searched the data sets of MI and T2DM from GEO. The results showed that 18 upregulated DEGs and four downregulated co-expressed DEGs were identified between MI and T2DM. We also performed GO 
Table 3 The Top 10 and Bottom 10 Chemical Compounds Identified by LI000 Platform

\begin{tabular}{|c|c|c|}
\hline Name & CMap Connectivity Score & Description \\
\hline IB-MECA & 99.93 & Adenosine receptor agonist \\
\hline Hydroxycholesterol & 99.61 & LXR agonist \\
\hline Morphothebaine & 99.61 & Adrenergic receptor antagonist \\
\hline Atovaquone & 99.6 & Mitochondrial electron transport inhibitor \\
\hline Tetrahydrocannabinol-7-oic-acid & 99.58 & Anti-inflammatory \\
\hline AG-370 & 99.48 & PDGFR receptor inhibitor \\
\hline Indapamide & 99.44 & Thiazide diuretic \\
\hline Etomoxir & 99.37 & Carnitine palmitoyltransferase inhibitor \\
\hline Diphenoxylate & 99.33 & Opioid receptor agonist \\
\hline Metixene & 99.33 & Acetylcholine receptor antagonist \\
\hline PU-H7I & -99.39 & HSP inhibitor \\
\hline Sulmazole & -99.47 & Adenosine receptor antagonist \\
\hline Ketoconazole & -99.61 & Sterol demethylase inhibitor \\
\hline Tipifarnib & -99.61 & Farnesyltransferase inhibitor \\
\hline Leucodin & -99.64 & Melanin inhibitor \\
\hline Niridazole & -99.75 & Phosphofructokinase inhibitor \\
\hline PD-169316 & -99.82 & p38 MAPK inhibitor \\
\hline Escitalopram & -99.82 & Selective serotonin reuptake inhibitor (SSRI) \\
\hline TUL-XXI039 & -99.89 & Serine/threonine kinase inhibitor \\
\hline PJ-34 & -99.93 & PARP inhibitor \\
\hline
\end{tabular}

enrichment and KEGG pathway enrichment analyses, and a PPI network was constructed to identify the top $10 \mathrm{hub}$ genes from among the common DEGs. There are eight coDEGs to demonstrate diagnostic value in MI and T2DM $(p<0.05)$, which may have an important role in early predict the risk of MI in T2DM.

Myeloperoxidase $(M P O)$, lactoferrin $(L T F)$, cathelicidin antimicrobial peptide (CAMP), azurocidin 1 (AUZ1), defensin alpha 4 (DEFA4) are expressed in the granules of the neutrophil, which participate in the progress of inflammatory reaction. $M P O$, an abundant heme-containing peroxidase, is mainly expressed in neutrophils and to a lesser degree in monocytes. ${ }^{19}$ In the presence of hydrogen peroxide and halides, MPO catalyzes the reactive oxide species including hypochlorous acid and hypohalous acid, which play an important role in inflammation and oxidative stress. ${ }^{20} M P O$ and its oxidative products have been found to be enriched in human atherosclerotic lesions where they co-localized with macrophages. $M P O$ and its end product $\mathrm{HOCl}$ could activate the MMPs and inhibit TIMPs, further promoting the reduction in the fibrous cap, and lead to the destabilized atherosclerotic plaque. ${ }^{21}$ In a large cohort of patients with chest pain, a single measurement of $M P O$ on admission independently predicted acute MI. Moreover, many studies demonstrated that MPO is significantly associated with follow-up cardiovascular events in MI patients. ${ }^{22,23}$ Furthermore, in a study assessing neutrophil activity in T2DM patients with and without cardiovascular diseases (CVD), researchers found that plasma $M P O$ concentration is elevated in those with CVD, suggesting plasma $M P O$ levels to be an additional biomarker of oxidative stress and cardiovascular risk in T2DM patients. ${ }^{24}$ In addition, LTF is a monomeric multifunctional iron-binding glycoprotein, with a single polypeptide chain of about 690 amino acid residues and two sialic acid molecules, which is mainly produced by neutrophils and epithelial cells. ${ }^{25}$ It has been reported that $L T F$ displays anti-inflammatory and antioxidant actions. ${ }^{26}$ It strongly inhibits cholesteryl ester accumulation from LDL by impairing its binding to macrophages and vascular smooth muscle cells. ${ }^{27}$ Though the regulating mechanism of $L T F$ on cardiovascular diseases is not totally clear, clinical study showed that increased baseline concentration of $L T F$ strongly predicated the long-term risk for fatal ischemic heart disease patients with newly diagnosed DM. $^{28}$ MPO and LTF are present in different granules, while their behavior as the biomarker of $\mathrm{MI}$ is equivalent. The specific granules containing $L T F$ are more readily mobilized than those containing $M P O$, which implies that $L T F$ may have a wider dynamic concentration range than $M P O{ }^{29}$ Once inside a lesion, neutrophils release granular proteins: $C A M P 1$ and $A Z U 1$ stimulate recruitment of 
inflammatory monocytes. During the pathogenesis of atherosclerosis, $A Z U 1$ is deposited on the endothelium and triggers monocyte arrest and upregulation of endothelial adhesion molecules. ${ }^{30}$ A clinical trial showed that the significant positive correlation of $A Z U 1$ with the SYNTAX score, Grace score and Killip class in MI patients, suggesting the strong association between inflammation and disease severity. ${ }^{31}$ The mechanism remains unclear and demands further investigation. CAMP (also named as LL-37) is produced in the atherosclerotic lesions, where it may function as an immune modulator by activating adhesion molecule and chemokine expression, thus increasing innate immunity in atherosclerosis. ${ }^{32}$ The relationship of the CAMP in destabilization of atherosclerotic plaque and MI remains unknown. DEFA was the family of human neutrophil peptides, which was familiar with DEFA1-3. DEFA1-3 has been confirmed as a risk factor of atherosclerosis and coronary artery disease. ${ }^{33}$ DEFA4 was encoded as human neutrophil peptides 4, while the role of DEFA4 in atherosclerosis and MI has not been investigated. The role of CAMP, AUZ1 and DEFA4 in T2DM or MI remains to be further studied.

Matrix metalloproteinase-9 (MMP9) belongs to the MMP family, which is involved in matrix degradation. $M M P 9$ has been found to be participated in the pathogenesis of diabetes and diabetic complications, including diabetic retinopathy. ${ }^{34}$ Moreover, it has been demonstrated that increased $M M P 9$ expression after MI exacerbates ischemia-induced chronic heart failure through regulating autophagic flux. ${ }^{35}$ MMP9 expression and activity were increased in the fibrous cap of atherosclerotic plaques, which promotes extracellular matrix (ECM) degradation and enhances inflammation cell infiltration. ${ }^{36}$ In addition, $M M P 9$ was reported to influence the stability of plaques via promoting the migration and proliferation of vascular smooth muscle cells (VSMCs). VSMCs can further secrete vascular endothelial growth factor (VEGF), which plays an important role in neovascularization (a risk factor for plaque instability). ${ }^{37}$ Circulating $M M P 9$ has been recognized as a biomarker of predicting the plaque rupture ${ }^{38}$ and increased $M M P 9$ expression was related to the leukocyte numbers and left ventricular dimensions and impaired cardiac function. Signal transducer and activator of transcription 3 (STAT3) is an $89 \mathrm{KD}$ protein and composed of six functional domains. It is a signaling molecule and transcriptional factor that plays important role, which attributes to its genomic action that upregulates genes that anti-apoptotic, pro-angiogenic. ${ }^{39}$ STAT3 has been investigated to participate in the pathogenesis of atherosclerosis, including contributing to VSMCs proliferation, migration and phenotype transformation. Mitochondrial STAT3 is a regulator of electron transport chain and $\mathrm{Ca}^{2+}$ homeostasis and has an effect on the mitochondrial production of oxidative stress, therefore playing a crucial role in atherosclerosis. ${ }^{40}$ While whether STAT3 regulates the instability of atherosclerotic plaque remains unclear. STAT3-deficient mice are more susceptible to myocardial ischemia injury, show increased cardiac apoptosis and infarct size and have reduced cardiac function and survival. $^{41}$ Moreover, it has been demonstrated that $\mathrm{N}$-acetylcysteine prevented myocardial injury in diabetic mice through Jak2/STAT3 ${ }^{42}$ Platelet endothelial cell adhesion molecule-1 (PECAM1 or CD31), one member of the Ig gene superfamily, is composed of six extracellular Ig folds. ${ }^{43} \mathrm{CD} 31$ was reported to play a protective role in the atherosclerotic plaque. It can limit the unwanted collateral inflammatory damage and regulating leukocyte migration. ${ }^{44} \mathrm{CD} 31$ can also reduce the leakage and intraplaque hemorrhage from plaque neovessels. ${ }^{45}$ Studies showed that the administration of antibodies against CD31 before reperfusion can reduce the infarct size. ${ }^{46}$ A study population of cross-sectional analysis that consisted of 420 subjects with T2DM lasting more than 10 years showed that 142 patients with MI and 310 patients with no CVD. There were significant differences of PECAM1 genotype distribution in patients with MI compared with subjects in the control group, which suggest that polymorphism of PECAM1 (C373G) may be a genetic marker of MI in T2DM patients. ${ }^{47}$

Several limitations existed in our study. Firstly, our results in this study mainly derived from microarray analysis that based on gene expression value, which may be not directly equivalent to the protein expression. Assay of polymerase chain reaction and microarray chip may be better for assessing the risk of T2DM related MI. Secondly, validation should be carried out both in vitro and in vivo.

In conclusion, the hub co-DEGs of $M P O, M M P 9$, CAMP, LTF, AZU1, DEFA4, STAT3, and PECAM1 may link T2DM and MI, which might provide an insight into potential novel biomarkers for improving the prognosis of MI related to T2DM.

\section{Abbreviations}

AUC, area under the curve; AUZ1, azurocidin 1; BP, biological processes; CAMP, cathelicidin antimicrobial 
peptide; CC, cellular components; CVD, cardiovascular diseases; DEFA4, defensin alpha 4; DEGs, differentially expressed genes; DM, diabetes mellitus; ECM, extracellular matrix; FC, fold change; GEO, Gene Expression Omnibus; GO, Gene ontology; KEGG, Kyoto Encyclopedia of Genes and Genomes; LTF, lactoferrin; MF, molecular function; MI, myocardial infarction; $M M P$, matrix metalloproteinase-9; $M P O$, myeloperoxidase; PECAM1, platelet endothelial cell adhesion molecule-1; PPI, protein-protein interaction; ROC, receiver operating characteristic; STAT3, signal transducer and activator of transcription 3; VEGF, vascular endothelial growth factor; VSMC, vascular smooth muscle cell.

\section{Data Sharing Statement}

All data generated or analyzed during this study are included in this published article.

\section{Funding}

This work was supported by the National Nature Science Foundation of China (No. 82000301).

\section{Disclosure}

The authors report no conflicts of interest in this work.

\section{References}

1. Pillon NJ, Loos RJF, Marshall SM, Zierath JR. Metabolic consequences of obesity and type 2 diabetes: balancing genes and environment for personalized care. Cell. 2021;184(6):1530-1544.

2. Magliano DJ, Chen L, Islam RM, et al. Trends in the incidence of diagnosed diabetes: a multicountry analysis of aggregate data from 22 million diagnoses in high-income and middle-income settings. Lancet Diabetes Endocrinol. 2021;9(4):203-211.

3. Mekhaimar M, Dargham S, El-Shazly M, Al Suwaidi J, Jneid H, Abi Khalil C. Diabetes-related cardiovascular and economic burden in patients hospitalized for heart failure in the US: a recent temporal trend analysis from the National Inpatient Sample. Heart Fail Rev. 2021;26(2):289-300.

4. Perveen S, Shahbaz M, Ansari MS, Keshavjee K, Guergachi A. A hybrid approach for modeling Type 2 diabetes mellitus progression. Front Genet. 2019;10:1076.

5. Huang JX, Liao YF, Li YM. Clinical features and microvascular complications risk factors of early-onset Type 2 diabetes mellitus. Curr Med Sci. 2019;39(5):754-758.

6. Davidson JA. SGLT2 inhibitors in patients with type 2 diabetes and renal disease: overview of current evidence. Postgrad Med. 2019;131 (4):251-260.

7. Zhang Y, Ding X, Hua B, et al. Predictive effect of triglycerideglucose index on clinical events in patients with type 2 diabetes mellitus and acute myocardial infarction: results from an observational cohort study in China. Cardiovasc Diabetol. 2021;20(1):43.

8. Tajik AA, Dobre D, Aguilar D, et al. A history of diabetes predicts outcomes following myocardial infarction: an analysis of the 28771 patients in the high-risk MI database. Eur J Heart Fail. 2017;19 (5):635-642.
9. Murcia AM, Hennekens CH, Lamas GA, et al. Impact of diabetes on mortality in patients with myocardial infarction and left ventricular dysfunction. Arch Intern Med. 2004;164(20):2273-2279.

10. Potier L, Roussel R, Zeller M, et al. Chronic kidney disease, diabetes, and risk of mortality after acute myocardial infarction: insight from the FAST-MI program. Diabetes Care. 2020;43(3):e43-e44.

11. Fillmore N, Mori J, Lopaschuk GD. Mitochondrial fatty acid oxidation alterations in heart failure, ischaemic heart disease and diabetic cardiomyopathy. Br J Pharmacol. 2014;171(8):2080-2090.

12. Schilling JD. The mitochondria in diabetic heart failure: from pathogenesis to therapeutic promise. Antioxid Redox Signal. 2015;22 (17):1515-1526.

13. Cui J, Liu Y, Li Y, Xu F, Liu Y. Type 2 diabetes and myocardial infarction: recent clinical evidence and perspective. Front Cardiovasc Med. 2021;8:644189.

14. Dahl-Jorgensen K, Larsen JR, Hanssen KF. Atherosclerosis in childhood and adolescent type 1 diabetes: early disease, early treatment? Diabetologia. 2005;48(8):1445-1453.

15. Poznyak A, Grechko AV, Poggio P, Myasoedova VA, Alfieri V, Orekhov AN. The diabetes mellitus-atherosclerosis connection: the role of lipid and glucose metabolism and chronic inflammation. Int J Mol Sci. 2020;21:5.

16. Zheng Y, Ley SH, Hu FB. Global aetiology and epidemiology of type 2 diabetes mellitus and its complications. Nat Rev Endocrinol. 2018;14(2):88-98.

17. Lee YB, Han K, Kim B, et al. Risk of early mortality and cardiovascular disease according to the presence of recently-diagnosed diabetes and requirement for insulin treatment: a nationwide study. J Diabetes Investig. 2021. doi:10.1111/jdi.13539

18. Lejay A, Fang F, John R, et al. Ischemia reperfusion injury, ischemic conditioning and diabetes mellitus. J Mol Cell Cardiol. 2016;91:11-22.

19. Loria V, Dato I, Graziani F, Biasucci LM. Myeloperoxidase: a new biomarker of inflammation in ischemic heart disease and acute coronary syndromes. Mediators Inflamm. 2008;2008:135625.

20. Ikitimur B, Karadag B. Role of myeloperoxidase in cardiology. Future Cardiol. 2010;6(5):693-702.

21. Teng N, Maghzal GJ, Talib J, Rashid I, Lau AK, Stocker R. The roles of myeloperoxidase in coronary artery disease and its potential implication in plaque rupture. Redox Rep. 2017;22(2):51-73.

22. Kolodziej AR, Abo-Aly M, Elsawalhy E, Campbell C, Ziada KM, Abdel-Latif A. Prognostic role of elevated myeloperoxidase in patients with acute coronary syndrome: a systemic review and meta-analysis. Mediators Inflamm. 2019;2019:2872607.

23. Dullaart RP, Tietge UJ, Kwakernaak AJ, Dikkeschei BD, Perton F, Tio RA. Alterations in plasma lecithin: cholesterolacyltransferase and myeloperoxidase in acute myocardial infarction: implications for cardiac outcome. Atherosclerosis. 2014;234(1):185-192.

24. Katakami N, Kaneto H, Matsuoka TA, et al. Accumulation of gene polymorphisms related to oxidative stress is associated with myocardial infarction in Japanese type 2 diabetic patients. Atherosclerosis. 2010;212(2):534-538.

25. Baker EN, Baker HM. Molecular structure, binding properties and dynamics of lactoferrin. Cell Mol Life Sci. 2005;62(22):2531-2539.

26. Baker EN, Baker HM. A structural framework for understanding the multifunctional character of lactoferrin. Biochimie. 2009;91(1):3-10.

27. Mayeur S, Spahis S, Pouliot Y, Levy E. Lactoferrin, a pleiotropic protein in health and disease. Antioxid Redox Signal. 2016;24 (14):813-836.

28. Vengen IT, Dale AC, Wiseth R, Midthjell K, Videm V. Lactoferrin is a novel predictor of fatal ischemic heart disease in diabetes mellitus type 2: long-term follow-up of the HUNT 1 study. Atherosclerosis. 2010;212(2):614-620.

29. Aminian A, Boudjeltia KZ, Babar S, et al. Coronary stenting is associated with an acute increase in plasma myeloperoxidase in stable angina patients but not in patients with acute myocardial infarction. Eur J Intern Med. 2009;20(5):527-532. 
30. Gaul DS, Stein S, Matter CM. Neutrophils in cardiovascular disease. Eur Heart J. 2017;38(22):1702-1704.

31. Ipek E, Yolcu M, Yildirim E, et al. A novel marker of inflammation: azurocidin in patients with st segment elevation myocardial infarction. Int J Mol Sci. 2018;19:12.

32. Edfeldt K, Agerberth B, Rottenberg ME, et al. Involvement of the antimicrobial peptide LL-37 in human atherosclerosis. Arterioscler Thromb Vasc Biol. 2006;26(7):1551-1557.

33. Maneerat Y, Prasongsukarn K, Benjathummarak S, Dechkhajorn W, Chaisri U. Increased alpha-defensin expression is associated with risk of coronary heart disease: a feasible predictive inflammatory biomarker of coronary heart disease in hyperlipidemia patients. Lipids Health Dis. 2016;15:117.

34. Aghadavod E, Soleimani A, Amirani E, et al. Comparison between biomarkers of kidney injury, inflammation, and oxidative stress in patients with diabetic nephropathy and Type 2 diabetes mellitus. Iran J Kidney Dis. 2020;14(1):31-35.

35. Nandi SS, Katsurada K, Sharma NM, Anderson DR, Mahata SK, Patel KP. MMP9 inhibition increases autophagic flux in chronic heart failure. Am J Physiol Heart Circ Physiol. 2020;319(6):H1414-H1437.

36. Jiang XB, Yuan WS, Wang JS, Liu Z, Liu DH, Shi ZS. Matrix metalloproteinase-9 expression in carotid atherosclerotic plaque and contrast-enhanced MRI in a swine model. J Neurointerv Surg. 2014;6 (1):24-28.

37. Papalambros E, Sigala F, Georgopoulos S, et al. Vascular endothelial growth factor and matrix metalloproteinase 9 expression in human carotid atherosclerotic plaques: relationship with plaque destabilization via neovascularization. Cerebrovasc Dis. 2004;18(2):160-165.

38. Chen F, Eriksson P, Hansson GK, et al. Expression of matrix metalloproteinase 9 and its regulators in the unstable coronary atherosclerotic plaque. Int J Mol Med. 2005;15(1):57-65.
39. Hirano T, Ishihara K, Hibi M. Roles of STAT3 in mediating the cell growth, differentiation and survival signals relayed through the IL-6 family of cytokine receptors. Oncogene. 2000;19(21):2548-2556.

40. Chen Q, Lv J, Yang W, et al. Targeted inhibition of STAT3 as a potential treatment strategy for atherosclerosis. Theranostics. 2019;9(22):6424-6442.

41. Samidurai A, Roh SK, Prakash M, et al. STAT3-miR-17/20 signalling axis plays a critical role in attenuating myocardial infarction following rapamycin treatment in diabetic mice. Cardiovasc Res. 2020;116 (13):2103-2115.

42. Lin J, Wang T, Li Y, et al. N-acetylcysteine restores sevoflurane postconditioning cardioprotection against myocardial ischemia-reperfusion injury in diabetic rats. $J$ Diabetes Res. 2016;2016:9213034.

43. Nourshargh S, Krombach F, Dejana E. The role of JAM-A and PECAM-1 in modulating leukocyte infiltration in inflamed and ischemic tissues. J Leukoc Biol. 2006;80(4):714-718.

44. Caligiuri G. CD31 as a Therapeutic target in atherosclerosis. Circ Res. 2020;126(9):1178-1189.

45. Newman PJ, Newman DK. Signal transduction pathways mediated by PECAM-1: new roles for an old molecule in platelet and vascular cell biology. Arterioscler Thromb Vasc Biol. 2003;23 (6):953-964.

46. Serebruany VL, Gurbel PA. Effect of thrombolytic therapy on platelet expression and plasma concentration of PECAM-1 (CD31) in patients with acute myocardial infarction. Arterioscler Thromb Vasc Biol. 1999;19(1):153-158.

47. Reschner H, Milutinovic A, Petrovic D. The PECAM-1 gene polymorphism - a genetic marker of myocardial infarction. Cent Eur J Biol. 2009;4(4):515-520.
International Journal of General Medicine

\section{Publish your work in this journal}

The International Journal of General Medicine is an international, peer-reviewed open-access journal that focuses on general and internal medicine, pathogenesis, epidemiology, diagnosis, monitoring and treatment protocols. The journal is characterized by the rapid reporting of reviews, original research and clinical studies

\section{Dovepress}

across all disease areas. The manuscript management system is completely online and includes a very quick and fair peer-review system, which is all easy to use. Visit http://www.dovepress.com/ testimonials.php to read real quotes from published authors. 